\title{
Integer Programming Model for Ship Loading Management
}

\section{A Case Study from Cement Industry}

\author{
João Fonseca (1), Ricardo Alves (1), Ana Regina Macedo (10, \\ José António Oliveira (1), Guilherme Pereira (1), and Maria Sameiro Carvalho(i) \\ ALGORITMI Research Center, University of Minho
}

\begin{abstract}
Industries only survive in the modern business world if they are prepared to improve processes whenever it is necessary. Therefore, companies need to adapt and/or modify processes to improve the level of service and reduce needless costs. In this article the logistics problem of planning a ship load operation of a Portuguese cement company is addressed. The objective is to determine the best way to transport the bagged cement from the warehouse to a ship reducing costs and optimizing forklifts operations. A mathematical programming model is proposed showing this methodology as a powerful tool to provide effective support to a more intelligent decision process.
\end{abstract}

Keywords: operations research, storage yard management, logistics optimization, cement industry, repositioning model.

\section{Introduction}

With the integration of technology in industrial systems, there is an increasing need, for companies, to stay updated and alert or else they will not survive in today's current competitive world.

The Cement Industry (CI) is the focus in this paper and in fact the Supply Chain Management (SCM) in the CI is a topic with limited research. Studies reflect that the three major concerns of the CI are the manufacturing process, the cement material management and sustainability [1]. While topics such as distribution and transportation were also studied [1], there is not much information about it. This work aims to contribute to these two main topics, with the optimization of the transportation of the products from the warehouses to the ship. More specifically, the logistics operations in the quay cranes area, that includes the quay cranes and the storage yard [3].

The quay crane's area, is a critical area to any industry because it is the vital link between the company and the international market. This specific area of any industry's plant or container terminal should be efficient otherwise this link will be at risk [4]. That is, the satisfaction of the final client can be damaged, if anything goes wrong in the process to deliver the goods in perfect conditions and in time. 
Cement is a cheap but heavy product and therefore transportation costs are higher compared to other variable costs in the industry [2]. This means that the operation of transportation has a large impact on a cement company and an effective planning and a good use of transportation resources are extremely important in this type of industry.

This paper describes a new approach to organize and manage the transport operations between the cement production area and the quay crane area, which is a crucial node in the Cement Industry's supply chain.

In the next sections of the paper a detailed explanation of the problem scenario and its particularities are going to be approached. The main goal of this study is to transform the current situation into a new optimized one, in order to reduce costs and improve the workflow.

\section{Problem Description}

The study involved a CI plant in Portugal. This company has a plant with a manufacture center and a warehouse near a quay. In this plant there was no coordination or planning for the process of transportation of the packets of bagged cement between the warehouse and the quay cranes that directly supplied the ship. This operation brings big issues to the company, because if anything goes wrong it would put at risk the loading process of the ship, and cause delays that would lead to higher costs. These delays often occur if the cranes' rate is not respected and the crane must stop working. The cranes' rate is the maximum number of packets that a crane can load the ship, in each period.

The bags of cement are usually grouped in large packets of about 50 small cement bags. Each of these large packets has about $2000 \mathrm{~kg}$ of cement. Each order usually asks for 5 to 6 thousand packets. The packets of cement are stored outside in the storage yard, where they will be waiting to be picked up and transferred close to the quay cranes.

\subsection{Current Situation}

The existing process starts with a randomly distribution of the packets of cement in the storage yard. Then, each distributed zone is transported to the quay crane, using the forklifts. After ending the transportation of the first zone, they go to the next adjacent zone, and transport the packets that are placed in there. They do this recurrently, until they finished loading the ship. This process takes, in average, 48 working hours, divided in shifts, with three forklifts to do the work.

This first approach brings benefits and disadvantages. This way of transport makes it more unlikely to damage the packets of cement because in this case the products are moved two times at most. On the other hand, this method of transportation withdraws part of the storage yard's area and implies, in most cases, that the forklifts travel some great distances. These unnecessary distances that the forklifts must travel can lead to delays. In return, these delays can interfere with the amount of time the ship has to stay in the quay or even 
interfere with the crane's rate. These two consequences of the delays are of great importance to this problem because the company would have to deal with high costs associated to time losses.

\subsection{The Repositioning Idea}

To improve the management of the storage yard's area and with the goal of optimizing the transportation method stated so far, a different method was studied the repositioning method. With this approach it is intended to add some agility to the process in question and search a decrease of its working time.

After a first look over the available area for storing the packets, three distinct areas stood out, due to their distance to the crane. One, at the center, quite close to the quay cranes, and the other two - one in each side, left and right - that were further away from the crane. Being aware of the large distances between each area and the crane, as well as considering the forklifts' velocity, were the main reasons to look over the advantages of products' repositioning. In fact, it is much easier to keep up with the crane's rate if the products are closer [?]. The total yard storage considered consists of around 7000 square meters, where thousands of packets of cement, with roughly 1.5 square meters each, are to be distributed.

Aiming to improve the workflow and reduce the disorder inside the storage yard, aisles in the middle of each area were included. Although losing inventory space, these empty areas of about 15 meters of width, allow an easy and correct manoeuvring of the forklifts.

The central areas, designated $\mathrm{C} 1$ and $\mathrm{C} 2$, since they are closer to the crane they are the only ones that feed it directly. On the other hand, and regarding the method of repositioning, the cement stored in the side areas, the right ones

(designated R1, R2 and R3) and the left ones (designated L1 and L2), of the storage yard, is only transported to the central areas (not to the crane). This will work as a compensation/repositioning of the cement absent in the center due to its transport to the crane and then to the ship.

\section{Problem Formulation}

The case presented in the previous section was studied in a real situation and because of that, there are many constraints and singularities that were considered. First, it was important to organize the data relative to the dimensions of each storage area.

The distances between the central areas and the crane were measured - about $20 \mathrm{~m}$, between the center and the left areas the distance varied from 82.5 to $137.5 \mathrm{~m}$, and between the center and the right areas it varied between 40 to $110 \mathrm{~m}$.

Afterwards, it was necessary to study the average velocity of the forklifts. The forklifts can reach different velocities, depending on the distance they travel. Thus, the greater the distance, the higher the average velocity they reach. In this 
sense, it was considered an average velocity between the storage areas, being this average velocity between $7 \mathrm{~km} / \mathrm{h}$ and $15 \mathrm{~km} / \mathrm{h}$.

Once these calculations were concluded, it was possible to determine the total time associated to each movement cycle. Each forklift can transport two cement packets each time. Thus, four stages compose each forklift cycle: (1) the time of loading the cement packets, (2) the time travelling between a storage area and the destination, (3) the unloading of the cement packets and (4) the time travelling back to the origin point.

The corresponding values of each cycle remained in few minutes. Then, with each cycle time, and considering that only one forklift is transporting between two storage areas, it was possible to calculate the maximum number of packets of cement that can be transported in one hour. This value is higher between the central area and the crane - 90 packets in one hour per forklift. On the other hand, it reaches a minimum value between L1 and C1, corresponding to 58 packets per hour per forklift.

\section{Optimization Model}

As stated before, the optimization model aims to improve the process of transporting the packets to the ship. In the next sections, the objective function, the decision variables, and the associated constraints are going to be presented.

The Excel contains a solver that can handle this type of problems. It allows to build an integer linear programming model through tables and cells, keeping it simple and organized. However, this solver is subject to a limitation on the number of constraints and variables.

Therefore, a different tool was used to overcome these limitations. The FrontLine Solver is an Excel extension that uses Gurobi Optimization and allows to use unlimited variables and constraints. Besides that, this solver is open source and allows fast computing. With this powerful tool, and with the data calculated so far, the optimization model was implemented.

$$
\begin{gathered}
\min \sum_{j=1}^{3} d_{j} \\
d_{j}=\left|h_{j}-h_{\text {med }}\right| \\
f_{i j} \leq 2 \\
\sum_{i=1}^{7} f_{i j} \leq 5 \quad, \forall j \quad, \forall i \\
0.9 \cdot t_{i} \leq \sum_{j=1}^{24} p_{i j} \leq 1.1 \cdot t_{i}
\end{gathered}
$$




$$
\begin{aligned}
& \sum_{j=1}^{3} c_{j} \leq 4 \\
& \sum_{j=1}^{3} u_{i j}=1 \quad, \forall i \\
& 0 \leq n_{i j} \leq 900
\end{aligned}
$$

In (1), $h_{j}$ refers to the no. of working hours at shift $j$, the $h_{\text {med }}$ is the average working hours per shift, the $d_{j}$ the deviation from average working hours per shift, on shift $j$.This objective function tries to create an organized work environment, minimizing the deviation from an average number of hours per shift. These hours comprise the total work done by all forklifts at that shift. This aims that, in a perfect scenario, all the work shifts use, approximately, the same amount of time.

Aiming to limit the number of working hours and forklifts per hour and per storage area, constraints (3) and (4) were designed, where: $f_{i j}$ is the no. of forklifts operating in area $i$, at hour $j$.

The number of packets transported, at each hour, between each pair of storage areas, is of great importance too. As stated in (5), where the $p_{i j}$ equals the no. of packets transported from area $i$, at hour $j$ and $t_{i}$ is the no. of packets to be transported, from area $i$, the total number of packets, transported in each storage area, must be equal to the initial number of packets. However, equality constraints are too restrictive to this type of models - ending with exaggerated solving time, higher computer capacity required and, frequently, difficulty in finding feasible solutions. Thus a $10 \%$ deviation in the number of packets transported from each area was allowed, promoting the search for a feasible solution.

Aiming to minimize the number of hours to load the ship, it was verified that, part of the time, the number of packets arriving to the cranes' area was too big for one crane only. It is assumed that a crane can only handle 180 packets per hour and, allowing more packets in that area would only cause congestion. An optimization model does not have in consideration the workflow that a real case scenario must have. Thus, to overcome the dispersed usage of the second crane, it was necessary to implement the constraint presented in (6), where $c_{j}$ is the no. of cranes used, at shift $j$.

A requirement of the problem is that the crane must not pause during working hours. At the center, the storage areas $\mathrm{C} 1$ and $\mathrm{C} 2$ are directly related to the crane. Thus, they must be in constant flow, to keep up with its cycle. On the other hand, forklifts operating in L1, L2 and R1, R2, R3, are only moving packets to the central areas, making sure all material is transported and that $\mathrm{C} 1$ and $\mathrm{C} 2$, respectively, do not be running on empty, with no packets. In (7) it is imposed that, once the movement of packets starts, from one of the sides, the job of transporting the packets from that area must be completed, during only one shift. In this restriction $u_{i j}$ is 1 if there is transport, from area $i$, at shift $j$. 
The number of packets in the center $(\mathrm{C} 1$ and $\mathrm{C} 2)$ changes continuously decreasing whenever they are transported from the central areas to the crane (or near) and increasing whenever the packets are transported from the sides (L1, L2, R1, R2 and R3) to the center. The constraint in (8), where $n_{i j}$ is the no. of packets, in area $i$, at hour $j$, implies that the number of packets in each storage area must not be less than zero, nor greater than a specific upper limit.

\section{Results}

Applying the model introduced in the previous section, it was possible to determine the optimal solution, considering all the constraints. Several possible scenarios were tested, varying the number of forklifts and total hours utilized to complete the job. Although these instances lead to several solutions to the problem, in this section, only the most viable results are shown. During the results analysis, it is important to have in mind that, at present, the company is able to load the ship in about 48 working hours and with 3 forklifts.

\subsection{Computational Results}

In this instance, the number of available forklifts was increased to 5 , and the labour spectrum was decreased to only 24 hours, divided in 3 shifts of 8 hours. Although using, at maximum, 2 more forklifts, it is verified that the ship loading period can be reduced to half.

Besides all the advantages in the working time, there are improvements in the utilization of resources. In fact, with this solution, it is possible for the ship to stay much less time in the quay, which can be of great appeal for the client and the company itself. Also, keeping the cranes' cycle of 180 packets per hour is not an easy task, requiring a constant transportation of material to its area. By increasing the number of forklifts operating simultaneously, it is possible to keep some of them closer to the cranes' area, maintaining this fast cycle, imposed to the system. The results of the computational solution are detailed below.

The total number of machine hours, given by the model, was 38 per shift, in every shift. Achieving a null deviation to the mean value, a perfect score for the objective function was reached. To complete the job, it was necessary a total of 114 hours.

The total number of forklifts, working at the same time, does not exceed 5 and in each area, it does not exceed 2. As expected, there were some lot more operating hours in the center areas than in the sides, to keep up with the cranes' fast loading rate of 180 packets per hour. Therefore, while at lateral areas only 1 shift was used in each one as imposed by (7), at the center, forklifts were used in all the shifts.

With this configuration of working hours, it was possible to transport 5040 packets to the ship. As shown, this is an acceptable number, since in all areas the lower and upper limit from (5) were respected and the goal of 10 ton of cement 
packets was reached. Also, the minimum and maximum of packets allowed at each instant and area, imposed by (8), were respected.

In some periods, the number of packets arriving at the cranes area was more than it could handle. This could cause an accumulation of material and, that way, an extra crane was needed to load the excess. As forced by (6) these periods were concentrated in only one shift, to reduce costs associated with this extra resource. Thus, at shift one and three only one crane was utilized, while at the second shift, an extra crane was added.

\section{Conclusion and Future Work}

In this paper, a new method of load repositioning was tested, to improve the operations of loading a ship with huge orders of cement packets, using forklifts. To achieve such goal, an optimization model was built. This model successfully reduced the makespan of the job to $50 \%$ and allowed a better utilization of the available resources. It is important to refer that, to the best of our knowledge, this is the first paper tackling the repositioning method, modelling it and showing promising results.

Load repositioning method was proven to improve the flow of movements and reduce the chaos inside the plant, as well as the total number of working hours. In fact, this solution may be very helpful not only to the company - who sees its operating costs reduced - but also to the client, able to get the orders with no delays.

Methods such as load repositioning are normally set aside. In this paper a model for dealing with the referred problem is presented and the advantages are discussed. Also, it is important to refer that despite the optimization model being quite simple, it could have a huge impact in a company costs structure and in its client service level.

\section{References}

1. Agudelo, I.: Supply chain management in the cement industry. Massachusetts Institute of Technology (2009)

2. Christiansen, M., Fagerholt, K., Flatberg, T., Haugen, O., Kloster, O., Lund, E. H.: Maritime inventory routing with multiple products: A case study from the cement industry. European Journal of Operational Research, 208(1), 86-9. (2011)

3. Diabat, Ali and Theodorou, Effrosyni: An Integrated Quay Crane Assignment and Scheduling Problem Using Branch-and-Price. Computers and Industrial Engineering, 73, 115-123 (2014)

4. Türkoğullari, Y. B., Taşkin, Z. C., Aras, N., Altinel, I. K.: Optimal berth allocation, time-variant quay crane assignment and scheduling with crane setups in container terminals. European Journal of Operational Research, 254(3), 985-1001 (2016) 